\title{
Dynamic Electrothermal Simulation of Integrated Resistors at Device Level
}

\author{
Bjorn Vermeersch, Gilbert De Mey \\ Dept. of Electronics and Information Systems (ELIS), Ghent University, \\ Sint Pietersnieuwstraat 41, 9000 Gent, Belgium. \\ E-mail: bjorn.vermeersch@elis.ugent.be, demey@elis.ugent.be
}

\begin{abstract}
This paper presents the dynamic electrothermal simulation of a rectangular resistor integrated on a semiconductor substrate. Due to the temperature dependence of the electrical conductivity of the resistive sheet, self heating provokes a coupling between the electrical and thermal problem and gives rise to nonlinear phenomena. We introduce a time stepping iterative method to perform the calculations. The electrical and thermal solvers are based on FEM and Green's functions techniques respectively. An extensive dynamic analysis of the device will be presented. The results include heating and cooling curves, Nyquist plot (complex locus) of the thermal impedance, time constant spectrum and structure function. Comparisons with the linear case, i.e. a temperature independent resistor, are made and accompanied by analytical approximations if possible. One key observation is that the nonlinearity may easily be overlooked: its detection is only possible in particular characteristics.
\end{abstract}

\section{INTRODUCTION}

It is a well known fact that the electrical characteristics of many electronic devices vary according to the operating temperature. This is simply a reflection of the underlying temperature dependence of a whole range of semiconductor properties, influencing the internal behaviour. Therefore heating of a device, either self-induced or by neighbouring electronic elements, may change the electrical operation. In turn, the alteration of dissipated power leads to a new temperature distribution, thus establishing a coupling between electrical and thermal behaviour.

In multi-chip modules electrothermal analysis is a useful instrument to detect and characterise coupling effects between the different components. Various simulators have been reported in the literature [1-3] both for steady state and transient modes. Typically both electrical and thermal behaviour are represented by an equivalent compact network model. Such a technique allows for a SPICE-like solution of the problem. Electrothermal coupling is then established by iteration between the electrical and thermal solver until convergence is reached, as shown schematically in Fig. 1.

Recently, attention is being dedicated also to device level simulation, e.g. for characterisation of hot spots in transistors and interconnection lines [4,5]. Whereas multichip simulators usually consider one single network node for every device, the

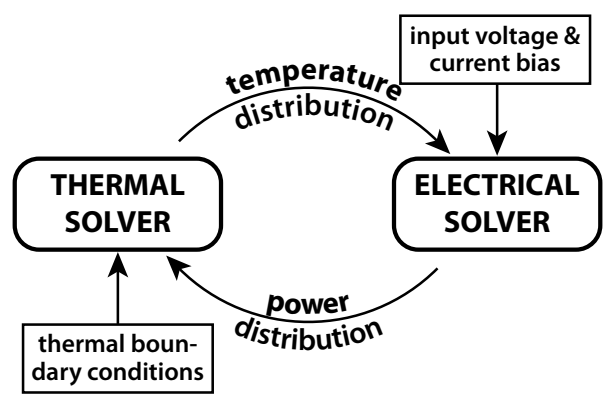

Fig. 1. Iteration diagram for electrothermal simulator.

current and power densities and temperature are actually nonuniformally distributed inside each component. This in itself gives rise to electrothermal coupling.

In this paper a dynamic electrothermal analysis will be carried out at device level for a single integrated resistor. In the next sections we will introduce the resistor design and describe the methods used in the electrical and thermal solver. Then a brief overview is given of the various methods and characteristics that can be used for dynamic thermal characterisation of the device. Next the simulation results are presented and discussed. Analytical approximations are provided for comparison; good matching with characteristics for 1-D spherical heat spreading is observed. The analyses clearly reveal nonlinearities caused by the electrothermal coupling. Finally a summary concludes the paper.

\section{INVESTIGATED STRUCTURE}

We will investigate a thin resistive film with thickness $d$ integrated on top of a semiconductor substrate. Electrodes are attached at two sides of the film, between which a voltage $V_{i n}(t)$ is applied. The considered layout is sketched in Fig. 2.

Although the method described later is in principle applicable to more complicated structures, this simple geometry is chosen to facilitate interpretation of the results and to enhance physical insight. A halfinfinite substrate is used mainly because of calculation time reasons. In principle however also finite 


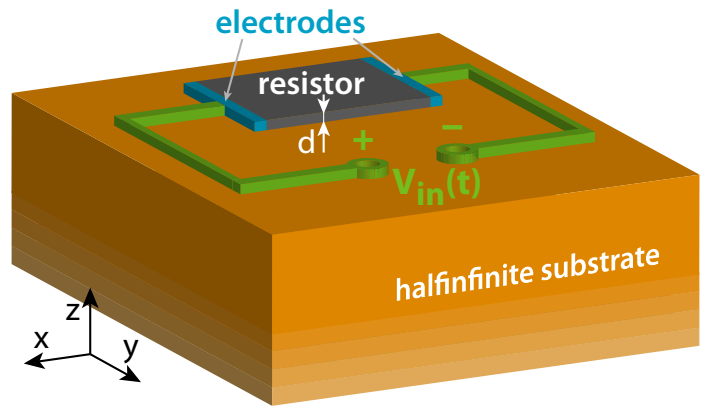

Fig. 2. Resistor integrated on halfinfinite substrate.

substrates or even multi-layered structures can be taken into account, as explained further in section III.B.

It would be interesting to apply the presented analysis method to a more realistic situation, e.g. a power transistor. The physical description for such a device will be quite different, in particular for the electrical model. Namely the Eqs. (1) - (5) need to be replaced by a coupled set of 'semiconductor equations' providing the carrier concentrations, electrical field, current density etc. In general terms however, phenomena similar to those observed in a resistor can be expected, e.g. the non-uniform temperature and power distributions could lead to current displacement and runaway behaviour.

\section{SiMULATOR PROCEDURES}

In order to simplify the modelling of the device, we will assume the thickness of the resistor to be much smaller than its lateral dimensions. In most practical situations this condition will be fulfilled. Due to this approximation all electrical and thermal fields inside the resistor can be treated as twodimensional, i.e. they are only $(x, y)$ dependent.

As a second key assumption we will suppose the resistor, from an electrical point of view, is operated in quasi-static regime. In other words it is assumed that for each change in the driving voltage the relaxation of all electrical phenomena is completely finished within the considered time step. In practice it can indeed be expected that electrical time constants are several orders of magnitude smaller than their thermal counterparts, except for very small devices. For high frequency operations, accurate electrical modelling would also require to take capacitive and inductive effects into account. This falls outside the scope of the current work. Here we simply assume the device is at every moment acting as a pure resistor.

\section{A. Electrical solver}

As explained earlier, the voltage distribution inside the resistor can be thought of as 2-D: $\phi=\phi(x, y ; t)$. The electric field $\vec{E}$ (in $\mathrm{V} / \mathrm{m}$ ) is then given by:

$$
\vec{E}(x, y ; t)=-\vec{\nabla} \phi(x, y, ; t)
$$

The resistor material is characterised by an electrical conductivity $\sigma(x, y ; t)$ (in $\Omega^{-1} \mathrm{~m}^{-1}$ ) and a temperature coefficient $\alpha$ (in $K^{-1}$ ). The latter describes a linearisation of the temperature dependency of the resistivity $\sigma^{-1}$, leading to:

$$
\sigma(T)=\frac{\sigma_{r e f}}{1+\alpha \Delta T}
$$

in which $\sigma_{r e f}=\sigma\left(T_{r e f}\right)$ is a reference value (typically at $300 \mathrm{~K})$ and $\Delta T=T-T_{r e f}$. As the temperature distribution is normally non-uniform, the conductivity is location dependent as well, hence the notation $\sigma(x, y ; t)$. The conductivity relates the electric field to the current density $\vec{J}$ (in $\mathrm{A} / \mathrm{m}^{2}$ ):

$$
\vec{J}(x, y ; t)=\sigma(x, y ; t) \vec{E}(x, y ; t)
$$

Under the assumed quasi-static regime, the continuity equation becomes:

$$
\vec{\nabla} \circ \vec{J}(x, y ; t)=-\frac{\partial \rho}{\partial t} \approx 0
$$

hence combining (1), (3) and (4) produces

$$
\vec{\nabla} \circ[\sigma(x, y ; t) \vec{\nabla} \phi(x, y ; t)]=0
$$

This is a partial differential equation (PDE) for the voltage inside the resistor. The boundary conditions are as follows: (i) $\phi(x, y ; t)=V_{i n}(t)$ at the positive electrode, (ii) $\phi(x, y ; t)=0$ at the negative electrode and (iii) $\frac{\partial \phi}{\partial n}=0$ along all other boundaries. Eq. (5) can be solved relatively easily using numerical techniques, such as FEM. A triangular mesh is generated and the voltage for each grid node is calculated. This was achieved with the PDEtools functions in Matlab. Once the voltage is determined, we can calculate the volumetric power density (in $\mathrm{W} / \mathrm{m}^{3}$ ) as:

$$
p_{V}(x, y ; t)=\vec{J}(x, y ; t) \circ \vec{E}(x, y ; t)
$$

The equivalent surface power density is then immediately obtained by multiplying $p_{V}$ with the thickness $d$ of the resistor. Finally this leads to:

$$
p(x, y ; t)=d \cdot \sigma(x, y ; t)\left[\left(\frac{\partial \phi}{\partial x}\right)^{2}+\left(\frac{\partial \phi}{\partial y}\right)^{2}\right]
$$

The obtained density $p$ functions as input for the thermal solver (see Fig. 1) to calculate the temperature distribution as explained below.

\section{B. Thermal solver}

The substrate material is characterised by a thermal conductivity $k$ (in $\mathrm{W} / \mathrm{mK}$ ), assumed isotropic, and a volumetric heat capacity $C_{v}$ (in $\left.\mathrm{J} / \mathrm{m}^{3} \mathrm{~K}\right)$. We will neglect the temperature dependence of $k$; one might however consider an a posteriori correction of the temperature field based on an estimated average temperature in the relevant substrate area [6]. Inside the substrate, the 3-D diffusion equation must be solved:

$$
k \nabla^{2} T(x, y, z ; t)-C_{v} \frac{\partial T}{\partial t}=0
$$

The resistor is dissipating power with a density $p(x, y ; t)$ as just obtained by the electrical solver. We will assume the adiabatic condition at the top substrate surface, hence all this power must be removed by conduction. A semi-analytical method will be used for temperature calculation, based on a Green's function. The latter is the fundamental solution of the heat equation, i.e. it gives the temperature field generated by a 1 Watt Dirac impulse both in space and time. The temperature generated by a distributed heat source as we are dealing with 
here is then found by superposition. In case of the halfinifinite substrate, we have for the source temperature:

$$
T_{s}(x, y ; t)=2 \int_{0}^{t} \mathrm{~d} \tau \iint_{\text {resistor }} p(x, y ; \tau) G(\Delta x, \Delta y, 0 ; t-\tau) \mathrm{d} x^{\prime} \mathrm{d} y^{\prime}
$$

where $\Delta x=x-x^{\prime}, \Delta y=y-y^{\prime}$ and $G$ is the Green's function for the 3-D space:

$$
G(\Delta x, \Delta y, \Delta z ; t)=\frac{\sqrt{C_{v}}}{8(\pi k t)^{3 / 2}} \exp \left(-\frac{C_{v} r^{2}}{4 k t}\right)
$$

with $r^{2}=(\Delta x)^{2}+(\Delta y)^{2}+(\Delta z)^{2}$. The extra factor 2 in (9) is needed because of the adiabatic boundary condition. Note that (9) can still be applied for substrates with finite thickness using a multiple reflection technique: one has simply to adjust the Green's function according to the image sources. The same holds for a squarely laterally limited substrate. For other cases, i.e. multiple layers, a different technique such as finite element or boundary element method must be used to obtain the temperature. For the actual calculation the resistor (heat source) is divided into a grid of rectangular elements. To each of them a power density is attributed according to the average values in the underlying triangular mesh of the electrical solver. The temperature for each rectangular segment is then found by numerical evaluation of the integral (9). Special care is taken for correct treatment of the singularities occurring in the Green's function. Once the thermal field is known, Eq. (2) produces the electrical conductivity needed by the electrical solver.

\section{Iteration and time stepping}

Let us consider a set of increasing time values $t=$ $t_{1}, t_{2}, \ldots, t_{i}, \ldots$ The time steps are chosen in such a way that they allow to accurately follow the input voltage. For each time value the aforementioned procedures are now alternated, i.e. we run the electrical / thermal / electrical / thermal / ... solver as illustrated in Fig. 1. The iteration continues until the relative change of a testing quantity, e.g. the average temperature, gets smaller than a preset value. At the end of each cycle the power density $p\left(x, y ; t_{i}\right)$ is stored in memory, since it is needed in (9). Then we move to the next time value, adjust the input voltage accordingly and start a new iteration cycle.

\section{DYNAMIC THERMAL CHARACTERISATION}

Before moving to the actual simulation results, we will briefly explain the various methods used to represent the dynamic thermal behaviour of electronic devices.

A first and obvious way is to provide the thermal step response. The power is suddenly switched either on or off and the according temperature is captured as a function of time. These responses, which are the heating and cooling curve respectively, are each others complement for a linear system. In case of electrothermal coupling however this equivalence is lost, as will be shown later.

A second characteristic is the thermal impedance. This is simply an extension of the well known thermal resistance $R_{t h}$ to dynamic phenomena. One may use a transient impedance $Z_{t h}(t)$, being the ratio of the temperature $T(t)$ and applied power $P(t)$, although the concept is more commonly used in the Laplace domain:

$$
Z_{t h}(s)=\mathcal{L}\left[Z_{t h}(t)\right]=\int_{0}^{\infty} \frac{T(t)}{P(t)} \exp (-s t) \mathrm{d} t
$$

The evaluation along the complex axis $Z_{t h}(s=j \omega)$ is particularly interesting, as this gives the thermal frequency response of the system. This function can be visualised as a Nyquist plot (complex locus), i.e. $\operatorname{Im}\left[Z_{t h}(j \omega)\right]$ vs. $\operatorname{Re}\left[Z_{t h}(j \omega)\right]$ with $\omega$ as a parameter.

Another way of representing the dynamic behaviour is the time constant spectrum. The heating curve normalised to a $1 \mathrm{~W}$ power step, i.e. the transient impedance, can be approximated as the superposition of a large number of exponentials of the form $R_{i}\left[1-\exp \left(-t / \tau_{i}\right)\right]$. In practice the thermal time constants are not limited to a discrete set of values $i$; they cover a wide range instead. So eventually the summation of discrete components merges into a continuous integral:

$$
Z_{t h}(t)=\int_{-\infty}^{\infty} R(\zeta)[1-\exp (-t / \exp (\zeta))] \mathrm{d} \zeta
$$

in which we used $\zeta=\ln (\tau)$ as a logarithmic time constant variable. The time constant spectrum $R(\zeta)$ can be obtained by numerical deconvolution of the differentiation of the step response in logarithmic time scale [7]. If the complex impedance function $Z_{t h}(s)$ is known, the spectrum can also be obtained as [7]:

$$
R(\zeta)=\frac{1}{\pi} \operatorname{Im}[Z(s=-\exp (-\zeta))]
$$

A final dynamic thermal characteristic we will use is the structure function. Two variants can be considered. The cumulative structure function (CSF) shows the cumulative thermal capacitance $C_{\sum}$ as a function of the cumulative thermal resistance $R_{\sum}$, while the differential structure function (DSF) uses $\mathrm{d} C_{\sum} / \mathrm{d} R_{\sum}$ as ordinate. Values for $R_{\sum}$ and $C_{\sum}$ are obtained from the elements in an equivalent Cauer network representing the dynamic behaviour. Such can be derived by turning the time constant spectrum into an equivalent Foster network, followed by a Foster-Cauer transformation. It has been shown that structure functions provide direct information about the heat flow path, where $R_{\sum}$ should be seen as a generalised coordinate [8]. One can e.g. prove the DSF is proportional to the squared cross section area of the heat flow path. This and other properties make the DSF a useful instrument for thermal characterisation and failure detection of multi-layered systems and electronic packages [8,9].

\section{Simulation Results}

We will study a $1 \mathrm{k} \Omega$ resistor design for a power amplifier (max. $15 \mathrm{~V}$ voltage drop over the resistor). The resistor is implemented as a $1 \mu \mathrm{m}$ thin film of n-type silicon with doping concentration of $10^{17} \mathrm{~cm}^{-3}$. This leads to [6]:

$$
\begin{aligned}
\sigma_{r e f} & =1.3 \times 10^{3} \Omega^{-1} \mathrm{~m}^{-1} \quad\left(R_{\text {sheet }}=769 \Omega / \text { square }\right) \\
\alpha & =0.0043 \mathrm{~K}^{-1}
\end{aligned}
$$


The resistor is integrated on a silicon substrate. The finally proposed design and all relevant electrical and thermal parameters are shown in Fig. 3.

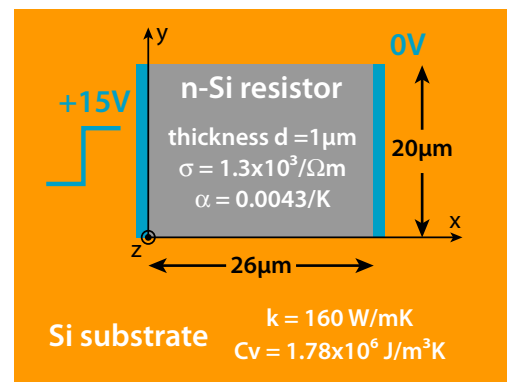

Fig. 3. $1 \mathrm{k} \Omega$ design used for electrothermal simulation.

We will analyse the behaviour during device switch-on (a voltage step of $+15 \mathrm{~V}$ is applied) at $t=0$. The resistor was divided into a mesh containing 3968 triangles and a grid of 520 squares for the electrical and thermal solver respectively. Results are to be compared with those found for a linear, temperature independent $(\alpha=0)$ resistor. The calculated heating curves were further processed using the T3ster software provided by MicRed company.

\section{A. Validation of the method}

First the proposed simulation technique is checked for selfconsistence. For this purpose we have calculated the average resistor temperature using different time step schemes (see Fig. 4).

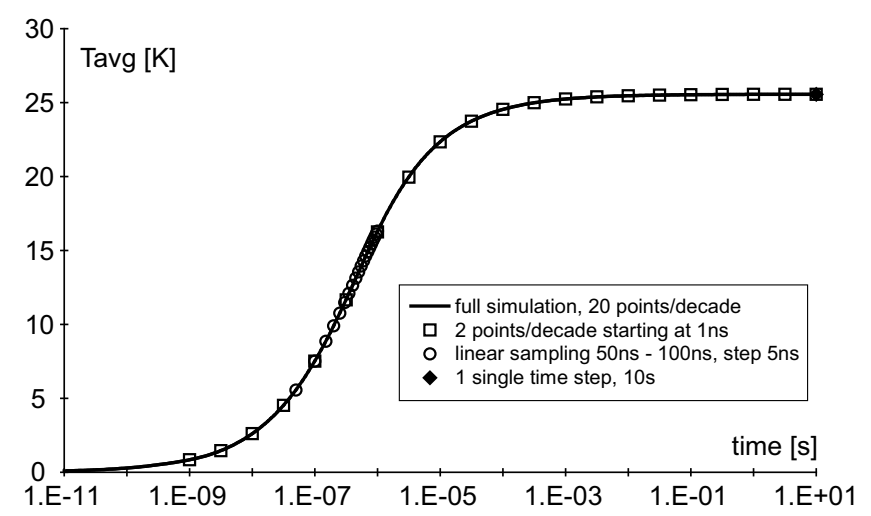

Fig. 4. Consistency check of the simulator.

One can clearly see that regardless the initial value or distribution of the time samples, the results are extremely similar. A very accurate estimation for the steady state value can be even obtained with one single time step simulation at $t=10 \mathrm{~s}$.

\section{B. Step responses}

Heating curves were simulated with a resolution of 20 points per decade. The obtained minimum, average and maximum temperatures are shown and compared with the linear case in Fig. 5. The graph also indicates the values obtained by a DC electrothermal simulator developed earlier [6].

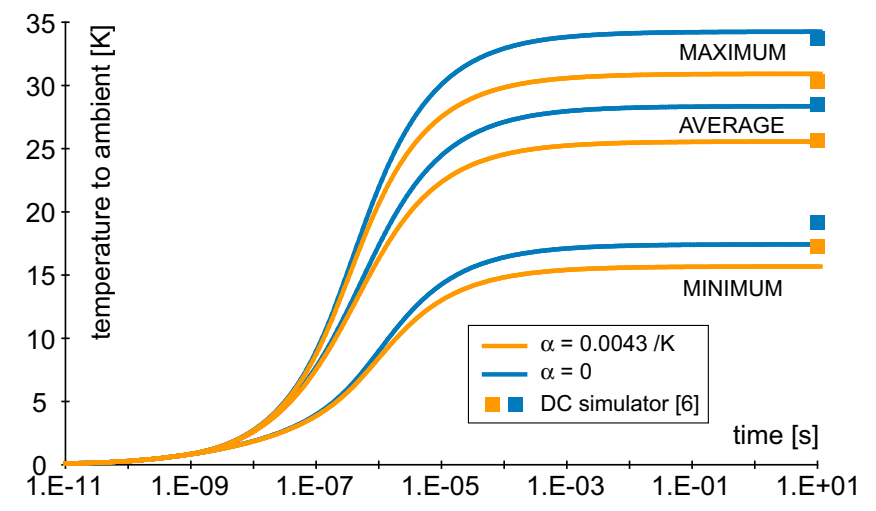

Fig. 5. Heating curves.

The simulators agree fairly well, except for the minimum temperature. The reason for this is not fully clear yet.

Initially all curves coincide, hence the temperature distribution is uniform. Moreover, up to about $200 \mathrm{~ns}$ both resistors are showing the same behaviour. It is only from that moment $\alpha$ starts to play a role and a deviation between the curves is observed. This can be easily explained. Initially the temperature rise is quite small. Hence the effective electrical resistance $R_{e f f}$, i.e. the input voltage divided by the total current, is close to the reference value. As the heating process continues and the temperature rises, the resistance will increase, which under a constant voltage reduces the dissipated power (see Fig. 6). This finally leads to temperatures that are lower compared to the linear case. It is needless to say that for the linear case both $R_{\text {eff }}$ and $P$ remain constant, at $1 \mathrm{k} \Omega$ and $225 \mathrm{~mW}$ respectively. Finally it was also observed the effective electrical resistance could be fitted extremely well using:

$$
R_{e f f}(t) \approx\left(1+\alpha T_{\text {avg }}(t)\right) \cdot R_{\text {ref }} \quad, \quad R_{\text {ref }}=1 \mathrm{k} \Omega
$$

as could intuitively be expected.

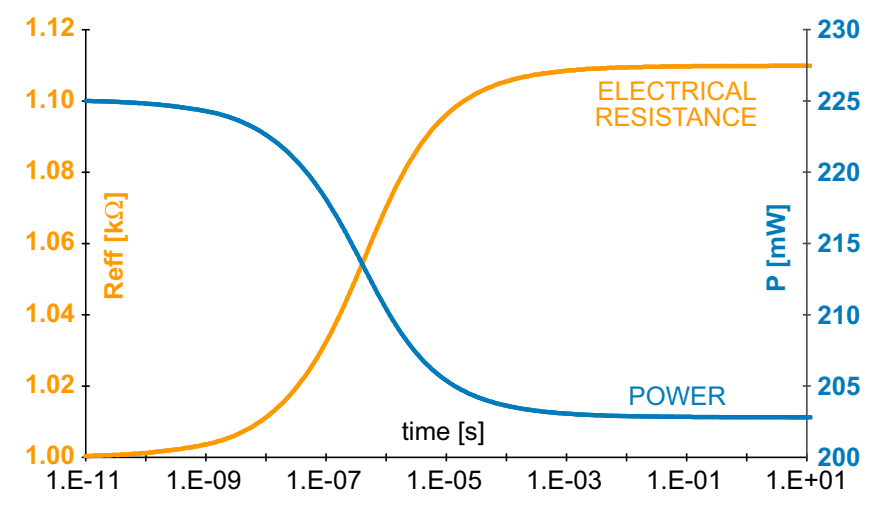

Fig. 6. Effective resistance and dissipated power.

\section{Thermal impedance}

Let us now calculate the thermal impedance, by normalizing the average temperature to the dissipated power $P(t)$. While the heating curves for $\alpha=0.0043$ and $\alpha=0$ were clearly different, their thermal impedance coincides both in the time and frequency domain (Figs. 7 and 8 respectively). 


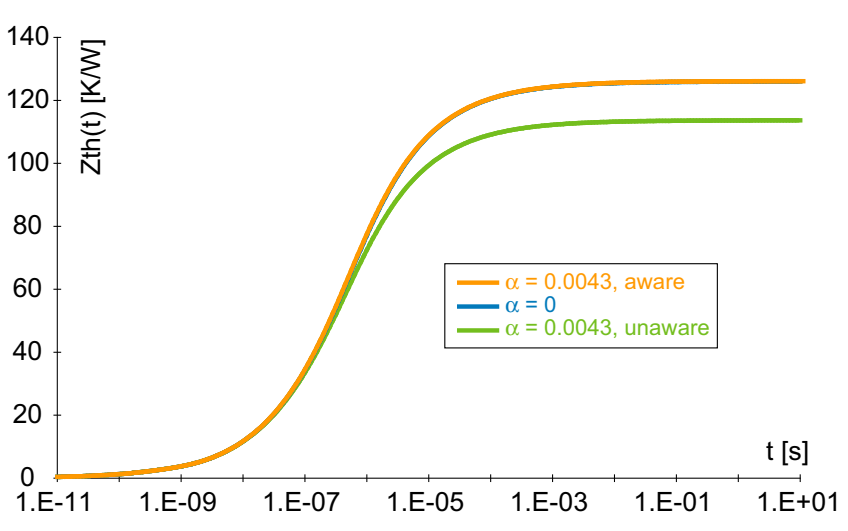

Fig. 7. Thermal impedance - transient curve.

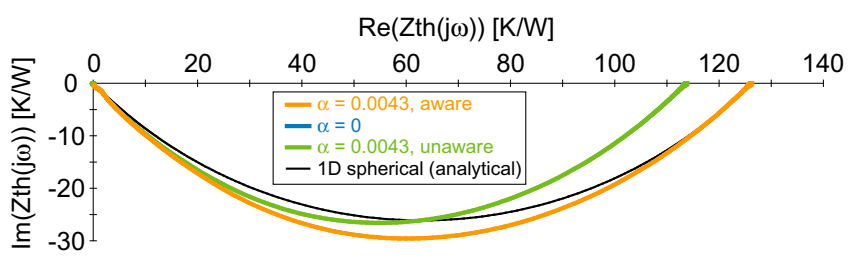

Fig. 8. Thermal impedance - Nyquist plot.

Based solely on the impedance plots, which are very commonly used as a major dynamic characteristic, the structures can simply not be distinguished. This suggests that $Z_{t h}$ is mainly determined by the resistor geometry and thermal substrate parameters. The minor influence that could arise from the altered temperature distribution is probably wiped out completely by taking the average temperature. In Fig. 8 the Nyquist plot corresponding to a 1-D spherical heat spreading is also given for comparison. For such a case one can prove analytically:

$$
Z_{t h}(s)=\frac{R_{t h}}{1+\sqrt{s / s_{0}}} \quad, \quad s_{0}=\frac{4 \pi^{2} k^{3} R_{t h}^{2}}{C_{v}}
$$

In the foregoing we took the variable nature of the power dissipation into account. When doing measurements in practice, we are however not always aware of the nonlinear behaviour of the device. One could erroneously assume that the voltage step simply leads to a constant power (namely $225 \mathrm{~mW}$ ) being applied to the resistor, and carry out the analysis accordingly. As can be seen in Figs. 7 and 8 (' $\alpha=0.0043$, unaware' curves), this approach leads to an underestimation of the thermal impedance.

\section{Time constant spectrum}

The transient impedance curves were further processed to obtain the time constant spectrum. The results are shown in Fig. 9. For time constants up to about $100 \mathrm{~ns}$ the spectra are almost identical. This should not be a surprise. It is again because initially only small temperature rises are generated, so nonlinear effects do not yet play a role. For larger $\tau$ values an interesting observation is made. Whereas the impedance seems identical for nonlinear and linear cases, small differences in the time constant spectrum are noticed.

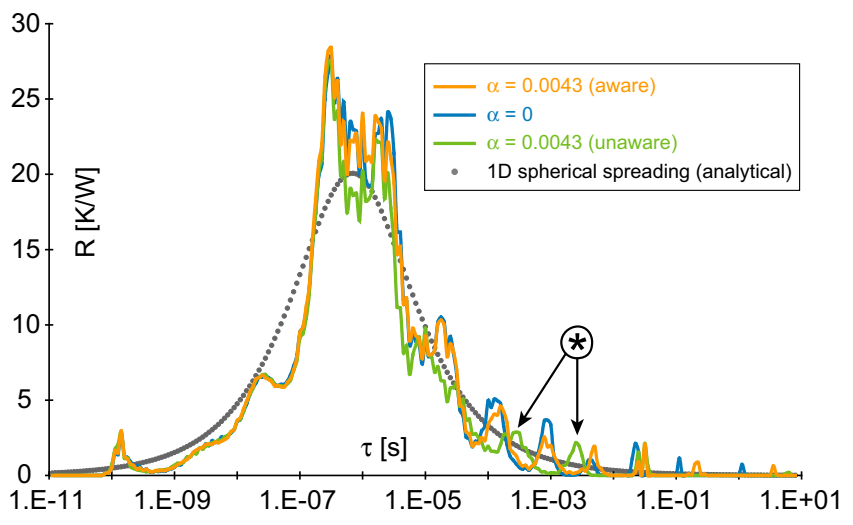

Fig. 9. Thermal time constant spectrum.

This illustrates the advantage of a multi-viewpoint analysis: some characteristics reveal information which cannot be detected in others. The difference between the two structures manifests itself in different sizes of certain peaks, however still at the same time scale. This is in contrast to the situation where one is not aware of the nonlinearity. In that case some peaks are even shifted, as indicated with (*) in Fig. 9.

The overall shape of the spectrum is predicted reasonably well by the result for spherical spreading. By applying (13) to (15) and going back to linear time scale, we find:

$$
R(\tau)=\frac{R_{t h}}{\pi} \frac{\sqrt{1 / s_{0} \tau}}{1+1 / s_{0} \tau}
$$

This function is also represented in Fig. 9 (dotted line).

\section{E. Structure function}

The differential structure function is shown in Fig. 10.

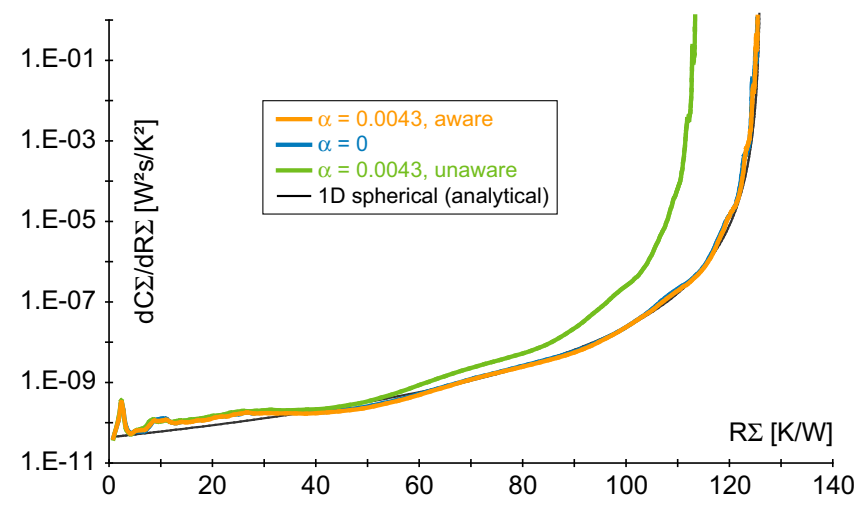

Fig. 10. Differential structure function.

We remind this function is strongly related to the heat flow profile. The alteration of the temperature distribution inside the resistor, caused by the nonlinearity, can be expected to have almost no influence on the way the heat spreads into the substrate. It is therefore not surprising that the structure functions for $\alpha=0.0043$ and $\alpha=0$ are nearly coinciding (see Fig. 10). When one is however not aware of the nonlinearity, the DSF is deviating, due to the underestimation of the thermal impedance. 
For a 1-D spherical heat flow, the structure function can be calculated analytically:

$$
\mathrm{DSF}=\frac{\mathrm{d} C_{\sum}}{\mathrm{d} R_{\sum}}\left(R_{\sum}\right)=\frac{C_{v}}{4 \pi^{2} k^{3}} \frac{1}{\left(R_{t h}-R_{\sum}\right)^{4}}
$$

This analytical model, also presented in Fig. 10, is very comparable to our results for $R_{\sum}>60 \mathrm{~K} / \mathrm{W}$. This means that sufficiently far away from the resistor the heat spreads almost radially in the substrate, as could be expected. The actual heat source is obviously a rectangle instead of half a sphere, explaining the less accurate fit close to the substrate surface (small $R_{\sum}$ ).

\section{F. Heating vs. cooling}

As a final illustration of the nonlinear behaviour we will dedicate some words on the cooling curve. Upon heating, the electrothermal coupling causes a continuous mutual influence between the power, current and temperature distributions. During cooling however the power is switched off, hence the coupling disappears. Apart from the different initial temperature distribution the resistor cools down just like a linear one. As a consequence, heating and cooling curves are not complementary when dealing with a temperature dependent resistor. This is clearly illustrated in Fig. 11: the summation of the two curves no longer gives rise to a constant value.

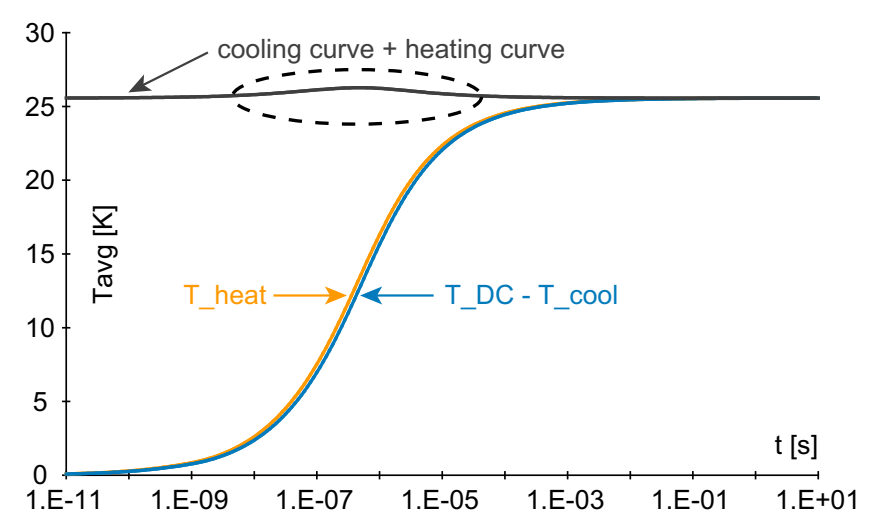

Fig. 11. Heating vs. cooling $(\alpha=0.0043)$.

\section{CONCLUSIONS}

We have presented a semi-analytical method that allows electrothermal simulation of integrated resistors. The studied example clearly illustrated non-linear effects. The calculated step response was further processed to obtain an extensive dynamic thermal characterisation. A key point to remember is that certain characteristics reveal more information than others: in some cases it was simply not possible to distinguish the resistor from a linear one. Finally the importance of being aware of potential non-linear behaviour should be stressed: assumption of a constant power application clearly leads to an incorrect characterisation of the device.

\section{ACKNOWLEDGMENTS}

Bjorn Vermeersch is preparing a $\mathrm{PhD}$ as a Research Assistant for the Research Foundation - Flanders (FWO - Vlaanderen) and would like to thank FWO for supporting the presented work.

\section{REFERENCES}

[1] Y.C. Gerstenmaier, A. Castellazzi, G. K. M. Wachutka, "Electrothermal simulation of multichip-modules with novel transient thermal model and time-dependent boundary conditions", IEEE Transactions on Power Electronics, Vol. 31, pp. 45-55, 2006.

[2] G. Buonaiuto, A. Irace, G. Breglio, P. Spirito, "THERMOS3, a tool for 3D electrothermal simulation of smart power MOSFETS", Proc. 12th THERMINIC, Nice (France), pp. 245-249, 27-29 September 2006.

[3] M. Kaminski, K. Slusarczyk, A. Napieralski, "Electrothermal simulation of analog and digital integrated circuits", Proc. 12th MIXDES, Krakow (Poland), pp. 249-254, 22-25 June 2006.

[4] F. Stefani, P.E. Bagnoli, "The hot-spot phenomenon and its countermeasures in bipolar power transistors by analytical electrothermal simulation", Proc. 12th THERMINIC, Nice (France), pp. 214-219, 27-29 September 2006.

[5] N. Spennagallo, L. Codecasa, D. D’Amore, P. Maffezzoni, ’Lumped electro-thermal model of on-chip interconnects", Proc. 12th THERMINIC, Nice (France), pp. 220-224, 27-29 September 2006.

[6] B. Vermeersch, G. De Mey, "Device level electrothermal analysis of integrated resistors", Proc. 14th MIXDES, Ciechocinek (Poland), pp. 375380, 21-23 June 2007.

[7] V. Székely, "Identification of RC networks by deconvolution: chances and limits", IEEE Transactions on Circuits and Systems, Vol. 45, pp. 244-258, 1998.

[8] V. Székely, T. Van Bien, "Fine structure of heat flow path in semiconductor devices: a measurement and identification method", Solid State Electronics, Vol. 31, pp. 1363-1368, 1988.

[9] V. Székely, "Enhancing reliability with thermal transient testing", Microelectron. Reliability, Vol. 42, pp. 629-640, 2002. 